$17^{\text {th }}$ International Congress of Metrology, 06003 (2015)

DOI: $10.1051 /$ metrology / 201506003

(C) Owned by the authors, published by EDP Sciences, 2015

\title{
Métrologie à l'EFS : de la production à la raison
}

\author{
REIFENBERG Jean Marc ${ }^{12}$ a, BEGUE Stéphane ${ }^{1}$ \\ ${ }^{1}$ Etablissement Français du sang, Services centraux - Direction Médicale, 20 avenue du Stade de France \\ 93218 La Plaine-Saint-Denis Cedex, France. \\ ${ }^{2}$ Etablissement Français du sang Pyrénées Méditerranée 392, avenue du Professeur Jean Louis Viala \\ 34184 Montpellier Cedex 4, France.
}

\begin{abstract}
Résumé. Pour répondre aux exigences réglementaires, et anticiper sur les besoins et référentiels de demain, l'EFS est engagé, depuis 2005, dans une démarche d'amélioration et de structuration de sa fonction métrologie. Pour cela, l'établissement a décidé d'internaliser les raccordements métrologiques pour les principales grandeurs critiques en transfusion (température, volumétrie), et de les faire reconnaitre par le COFRAC. Cette stratégie s'est ainsi traduite par une augmentation importante des activités d'étalonnage et d'essai en interne, organisées en partenariats entre établissements « clients » et laboratoires d'étalonnages et d'essais accrédités.
\end{abstract}

Les méthodes se sont ainsi améliorées et homogénéisées. Les performances des équipements soumis aux étalonnages et aux essais sont dorénavant mieux connues et maîtrisées; les stratégies d'investissement reposent sur des cahiers de charges de plus en plus précis. L'expertise technique et la maturité de la fonction métrologie nationale constituent aujourd'hui des atouts permettant de revoir de manière plus avertie la pertinence de certains raccordements et leurs périodicités associées. L'objectif de cette présentation est d'illustrer ces réflexions avec quelques exemples (enceintes thermostatiques, enregistreurs de températures...). La revue des besoins, l'exploitation des historiques métrologiques, l'analyse de la criticité des instruments, l'approche par les risques représentent des pistes d'amélioration et d'efficience de cette activité indispensable à notre établissement.

\section{Introduction}

\subsection{Présentation de l'établissement}

Composé de 17 établissements régionaux, l'établissement français du sang (EFS) gère les activités de collecte, de préparation, de qualification et de distribution des Produits Sanguins Labiles (PSL) et alimente plus de 1 900 établissements de santé partout en France. II est présent sur l'ensemble du territoire avec 152 sites de prélèvement et 40000 collectes mobiles sont organisées chaque année. Son activité principale concerne le don de sang, le don de plasma et le don de plaquettes. II

répond chaque année aux besoins d'un million de malades. L'établissement assure également l'approvisionnement en plasma du LFB (Laboratoire français du fractionnement et des biotechnologies), en vue de la fabrication de médicaments dérivés du sang.

L'EFS est aussi le plus important laboratoire de biologie médicale (LBM) de France. II a réalisé 527 millions de B en 2013 et dispose d'une expertise reconnue en immunohématologie receveurs et en immunogénétique. Au-delà de son cœur de métier, l'EFS mène des activités thérapeutiques et de recherche dans des domaines innovants comme la thérapie cellulaire et tissulaire.

\subsection{Organisation de la métrologie à l'EFS}

La structuration de la métrologie à l'EFS a débuté en 2005, suite à un état des lieux exhaustif de la fonction nationale. Dans un souci d'autonomie, et de capacité de maîtrise de ses grandeurs et équipements critiques, l'entreprise a décidé d'internaliser la majeure partie des étalonnages et des essais dans les domaines de la température et de la volumétrie. Outre les activités gérées de manière autonome par chaque région, des laboratoires 
d'étalonnage de référence, à vocation inter régionale, ont été créés pour mutualiser les compétences et les moyens d'étalonnages au service de l'établissement unique.

Actuellement, le volume d'activité national se chiffre à environ 24000 rapports d'étalonnages et d'essais internes par an ; de nombreuses activités d'étalonnage et d'essais sont dorénavant accréditées par le COFRAC (Figure 1).

D'un point de vue organisationnel, l'animation et la coordination de la fonction métrologie est assurée par les services centraux de l'EFS. Depuis quelques années, au sein de la cartographie nationale des processus, le processus métrologie est positionné en processus de surveillance indépendant du processus support de gestion des équipements. II est totalement intégré dans le système de management de la qualité de l'établissement. Outre la surveillance règlementaire, il est soumis également à un programme d'audits internes ambitieux.

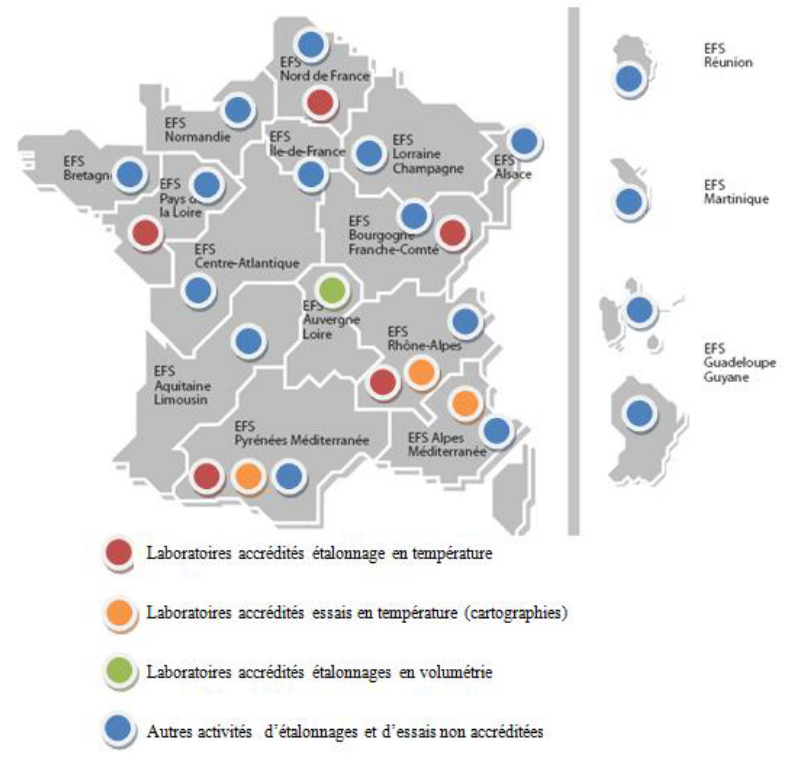

Figure 1 : organisation des activités d'étalonnage et d'essai à l'EFS

Cette organisation, le développement des compétences et le retour d'expérience sur des années d'activités de métrologie confère à l'EFS des atouts capitalisables. Les chapitres qui suivent illustrent quelques pistes techniques, stratégiques qui méritent d'être explorées pour optimiser la fonction.

\section{La surveillance des enceintes thermostatiques}

L'EFS Pyrénées Méditerranée (EFS-PYM), qui réalisait auparavant une cartographie de température annuelle de toutes les enceintes de la région a établi le constat, en 2008, qu'environ $90 \%$ des enceintes restaient conformes d'une année à l'autre. Ce constat a induit la remise en question de la stratégie de contrôle [1].

La quasi-totalité des enceintes à l'EFS est critique pour l'ensemble des activités médico techniques. D'un point de vue métrologique, cette criticité se caractérise par leur aptitude à conserver les produits, ou à réaliser des essais, dans un volume déterminé et dans des conditions de conformité exprimées en \pm EMT (Erreurs Maximales Tolérées). L'ensemble des points de la cartographie, et en particulier le point le plus chaud et le plus froid doivent donc demeurer en permanence à l'intérieur des tolérances.

La surveillance des performances des enceintes est effectuée, dans la quasi-totalité des cas grâce une sonde connectée à un dispositif enregistreur : disque, système de GTC (Gestion Technique Centralisée). Cette chaîne de mesure, indépendante du système de régulation, appelée témoin d'environnement selon FDX 15-140 [2], assure donc, en un seul point, la traçabilité de la température de conservation ou d'essai dans un espace plus ou moins volumineux, et la sécurité des produits grâce à un système d'alarmes de dépassement de seuils.

La solution métrologique classiquement rencontrée consiste à raccorder périodiquement l'enceinte (cartographie, suivie d'une déclaration de conformité aux EMT) et le témoin d'environnement (étalonnage, suivi d'une déclaration de conformité selon la règle du quart de l'EMT de l'enceinte) [3] ; les paramétrages des seuils d'alarme de la GTC sont ensuite corrigés en fonction des résultats d'étalonnage du témoin d'environnement.

Le traitement séparé des performances métrologiques de l'enceinte d'une part, et du témoin d'environnement d'autre part, amène néanmoins des questions. Du fait des biais d'homogénéité de l'enceinte, la réponse du capteur dépendra, en termes de traçabilité et de déclenchement d'alarme, de sa position dans la zone exploitée. La justesse de la mesure, et le report des données d'étalonnage sur les seuils d'alarme, n'auront de sens qu'à l'endroit où la sonde est positionnée. Mais l'utilisateur soucieux de la conformité de son enceinte est dans l'attente d'un indicateur lui permettant de suivre aussi l'homogénéité de son enceinte dans le temps.

II apparaitrait donc plus pertinent de caractériser la représentativité du témoin d'environnement et d'intégrer dans son suivi les caractéristiques d'homogénéité de l'enceinte à l'intérieur des EMT de conformité. Le fascicule de documentation FDV 08-601 [4], appliqué depuis des années à l'EFS-PYM, permet de suivre simultanément les deux paramètres (Figure 2). 


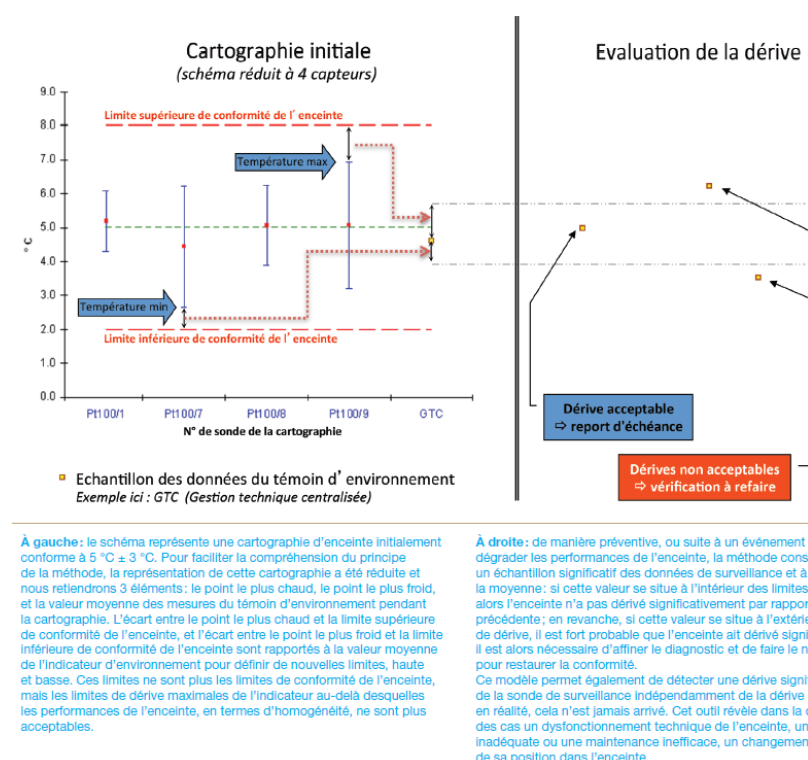

Figure 2 : Illustration du principe du fascicule de documentation FD V 08-601 [5]

Le principe de la méthode consiste à surveiller les performances des enceintes en termes d'homogénéité au travers du témoin d'environnement, avec des limites de dérive calculées à partir des EMT de l'enceinte et des valeurs d'homogénéité issues de sa caractérisation. Ainsi, une dérive significative de l'enceinte, mais aussi de la sonde de surveillance seront détectables. L'intérêt de cette méthode est qu'elle place le besoin de l'utilisateur au cœur de la stratégie, à savoir des conditions de stockage et d'essai conformes dans une zone identifiée. Elle permet de surveiller également les performances du témoin d'environnement, et ainsi de s'affranchir d'un étalonnage préventif systématique, sachant qu'après plusieurs années d'application du modèle, il s'avère dans la quasi-totalité des cas, les dérives confirmées provenaient des enceintes, et non pas de leur témoin d'environnement [1] [5].

Cette logique de raisonnement, qui consiste à rapprocher les caractéristiques des sondes de surveillance aux besoins, vaut aussi pour les sondes d'ambiance. Dans la majeure partie des cas, leur raccordement apparait peu pertinent dans la mesure où l'erreur de la sonde est le plus souvent négligeable par rapport aux EMT et aux biais d'homogénéité de la zone ambiante. Par exemple, la cartographie du laboratoire de métrologie de Montpellier, qui bénéficie de bonnes conditions de climatisation et de ventilation, a révélé une valeur d'homogénéité de $3,8{ }^{\circ} \mathrm{C}$ alors que l'incertitude des sondes de surveillance est inférieure à $0,5^{\circ} \mathrm{C}$. Comme pour les sondes qui surveillent les enceintes, et à plus forte raison dans ce contexte, un étalonnage de la chaîne de mesure permettra uniquement de quantifier la précision de la mesure en un point donné, et de justifier d'un raccordement pour répondre aux exigences. Alors qu'il est possible de raccorder aux étalons les témoins d'environnement des enceintes thermostatiques via les cartographies, cela n'est pas possible pour les zones d'ambiances qui ne sont en général pas caractérisées en température.

Ici, la logique du contrôle pourrait passer plus raisonnablement par une analyse de risque mettant en relation les spécifications d'ambiance à surveiller, et la position du témoin d'environnement en regard des zones les plus critiques.

\section{La périodicité d'étalonnage des} enregistreurs de température [6]

Dans le respect des procédures nationales, qui stipulent que "la périodicité d'étalonnage des chaînes de mesure de température mobiles est annuelle sauf exceptions validées et documentées ", le laboratoire de métrologie de l'EFS-PYM étalonne annuellement des enregistreurs de température "Logtag Logger » (société Waranet Solutions). Ces enregistreurs sont utilisés pour contrôler les températures de transport de Concentrés de Globules Rouges (CGR) et de Plasmas Frais Congelés (PFC).

Depuis quelques années, le pourcentage annuel d'enregistreurs de température nonconformes varie de 3 à $5 \%$ environ, la quasitotalité des non conformités se situant dans la gamme de températures négatives. Afin d'évaluer plus précisément les dérives à l'origine de ces non conformités, une étude expérimentale a été menée entre 2011 et 2013 , sur 572 enregistreurs, originaires de l'ensemble des sites de l'EFS PYM, prenant ainsi en compte les conditions d'utilisation dans l'échantillonnage.

Afin d'encadrer les températures d'emploi, chaque enregistreur a fait l'objet d'un premier étalonnage COFRAC en milieu aérien $\left(U_{\min }=\right.$ $0,4, k=2$ ), aux consignes $26{ }^{\circ} \mathrm{C}, 0^{\circ} \mathrm{C}$. Les instruments utilisés pour contrôler la température de transport des PFC $(\mathrm{N}=368)$ ont fait également l'objet d'un étalonnage à $-30^{\circ} \mathrm{C}$. Les déclarations de conformité ont été établies par rapport aux EMT définies dans les documents cadres nationaux, déduites de la règle du quart [3]. Après environ une année d'utilisation, chaque enregistreur a fait l'objet d'un nouvel étalonnage en l'état dans des conditions strictement identiques à celle de l'étalonnage initial. A chaque consigne de température, l'écart absolu entre les erreurs de justesse à l'année $\mathrm{n}$ et l'année $\mathrm{n}+1$ a été calculé (soit $d$ cette valeur). Les résultats sont consignés dans le tableau suivant (tableau $n^{\circ}$ 1).

\begin{tabular}{|l|c|c|c|}
\hline Consigne & $26^{\circ} \mathrm{C}$ & $0{ }^{\circ} \mathrm{C}$ & $-30^{\circ} \mathrm{C}$ \\
\hline Nb d'enregistreurs & 572 & 572 & 368 \\
\hline $\mathrm{d}(\max )$ & 0,3 & 0,4 & 1,0 \\
\hline
\end{tabular}




\begin{tabular}{|l|c|c|c|}
\hline $\mathrm{d}(\mathrm{min})$ & $-0,5$ & $-1,1$ & $-2,6$ \\
\hline $\mathrm{d}$ (moy) & 0,1 & 0,1 & 0,3 \\
\hline $\begin{array}{l}\text { Nb de non } \\
\text { conformes }\end{array}$ & 0 & 4 & 19 \\
\hline$\%$ de non conformes & $0,0 \%$ & $0,7 \%$ & $5,2 \%$ \\
\hline
\end{tabular}

Tableau 1: synthèse des résultats

Les résultats confirment que les dérives des enregistreurs de température n'impactent pas les déclarations de conformités dans la gamme de températures positives.

Ces dérives sont plus importantes dans la gamme de températures négatives. II convient néanmoins d'en relativiser les conséquences $(5,2 \%$ de NC annuels). En condition d'utilisation, si une dérive survenait soudainement lors d'un contrôle de transport de PFC, les conséquences de ce risque seraient très faibles : en effet, admettons que la température réelle se situe à la limite règlementaire, soit $-25^{\circ} \mathrm{C}$. D'après les résultats expérimentaux (dérive maximale de $2,6{ }^{\circ} \mathrm{C}$ ), le biais provoquerait empiriquement une traçabilité de $-22.4{ }^{\circ} \mathrm{C}$. Selon les procédures qui encadrent la conduite à tenir vis à vis de produits sanguins labiles exposés à des températures non conformes, " une exposition prolongée jusqu'à 2 mois à $-18^{\circ} \mathrm{C}, 1$ mois à $10^{\circ} \mathrm{C}$ ou $4 \mathrm{~h}$ à $+4^{\circ} \mathrm{C}$ n'altère pas significativement la qualité du plasma ». Les durées de transports régionaux étant inférieures à $24 \mathrm{~h}$, le risque de jeter un produit conforme, du seul fait d'une erreur de l'enregistreur, est extrêmement faible.

L'historique métrologique des enregistreurs non conformes dans la gamme négative a été examiné afin de vérifier si la conformité pouvait perdurer en l'état, sans opération d'ajustage, au-delà de 2 ans d'utilisation. Cet état des lieux révèle que la durée de validité des spécifications en température négative dépasse dans la majeure partie des cas deux années d'utilisation sans passer par des opérations d'ajustage intermédiaires parfois nécessaires pour corriger les dérives. D'un point de vue plus fonctionnel, tout dysfonctionnement ou dérive brutale, pouvant être généré par un défaut d'alimentation, notamment dans la gamme négative, est contrôlé par un témoin de charge qui alerte les utilisateurs lorsque la batterie est trop basse.

Compte tenu de ce retour d'expérience, et du risque encouru vis-à-vis les produits, l'EFS-PYM a décidé de passer d'une périodicité d'étalonnage annuelle de ces enregistreurs de température à une périodicité bisannuelle.

\section{Conclusion :}

A l'EFS, la métrologie internalisée assure depuis quelques années un suivi assidu des grandes catégories d'équipements (appareils volumétriques, chaînes de mesures de température, enceintes et bains thermostatés, surgélateurs, instruments de pesage, centrifugeuses ...). La compétence des unités de métrologie contribue à une meilleure expression des besoins métrologiques (cahiers de charges) etune évaluation des équipements et prestations critiques plus avertie.

La métrologie est sollicitée lorsque de nouveaux besoins apparaissent : changements règlementaires (spécifications métrologiques), évolutions techniques (nouveaux équipements). Malgré tout, bien que quelques études aient déjà été publiées $[7,8]$, les équipes de métrologie sont paradoxalement peu concertées dans le cadre de la validation de méthodes, alors que ces dernières sont parfois à l'origine de dépôts de brevets et de publications. Pourtant, la robustesse et la reproductibilité des travaux dépendent en grande partie de la caractérisation métrologique des procédés.

La course effrénée aux contrôles périodiques laisse en réalité peu de place à la réflexion et à l'optimisation. Pourtant l'entreprise a acquis depuis des années une réelle maturité ; elle a aussi accumulé de multiples données, malheureusement insuffisamment exploitées pour le compte de l'établissement unique. La finalisation de la structuration nationale des activités d'étalonnage, l'harmonisation des méthodes et des dispositions métrologiques vont se poursuivre pour gagner en cohérence et tendre vers l'efficience. Les outils de validation des méthodes, d'analyses de risques et d'exploitation statistique d'historiques métrologiques mériteront d'être plus utilisés pour mieux exprimer encore les besoins métrologiques et contrôler plus " utilement ».

Enfin, les atouts métrologiques de l'EFS doivent lui permettre de s'adapter au monde de demain : sa future organisation devra certes continuer à produire, le plus raisonnablement possible, des rapports d'étalonnages et d'essais de routine. Elle devra aussi anticiper sur l'évolution des produits et des référentiels de demain, et sur les besoins d'avenir comme la recherche et plus généralement les activités innovantes

\section{Références}

1. J.M. Reifenberg, E. Riout, A. Leroy. Surveillance des enceintes thermostatiques à l'établissement français du sang Pyrénées Méditerranée. Surveillance des Processus de mesure - Ou comment garantir au quotidien la performance des processus de mesure ? Coll. Tech. du CFM (mai 2009).

2. Fascicule de documentation FDX 15-140: Enceintes climatiques et thermostatiques: Caractérisation et vérification (2013). 
3. Document COFRAC SH GTA 01. Guide technique d'accréditation en biologie médicale (2015).

4. Fascicule de documentation FDV 08-601: Enceintes thermostatiques : Caractérisation, vérification et suivi quotidien (2005).

5. J.M. Reifenberg, E. Riout, A. Leroy. Métrologie dans un laboratoire de biologie médicale : enjeux et difficultés. Rev. Franc. Des Labo 461 // 69 (2014).

6. J.M. Reifenberg, E. Riout, A. Leroy, S. Begue. Métrologie à l'EFS : de la production à la raison. Transf. Clin. et Biol. 21 132-138 (2014).

7. O. Bouix, P. Sanchez, J.-M. Reifenberg. Définition des erreurs maximales tolérées pour les grandeurs physiques ayant un effet significatif sur le résultat du groupage $A B O-$ $\mathrm{RH} 1$ en technique microplaque. Transf. Clin. et Biol 14, 348-351 (2007).

8. L. Mannessier, M. Delamaire, O. Bouix, C. Krause, F. Roubinet. Maîtrise des tolérances métrologiques : application à la recherche des anticorps anti-érythrocytaires (RAI) en test indirect à l'antiglobuline (TIA) et au groupage sanguin $A B O$ par les procédés de filtration et en microplaque. Transf. Clin. et Biol 13, 271-277 (2006). 